\section{Taking another tilt at high secure hospitals}

\author{
The Tilt Report and its consequences for secure psychiatric \\ services $^{\dagger}$
}

TIM EXWORTHY and JOHN GUNN

The (second) Ashworth Inquiry (Fallon et al, 1999) recommended that 'an independent review of all aspects of physical security' at Ashworth (high secure) Hospital should be undertaken. This recommendation subsequently broadened to include all three high-security hospitals in England (Ashworth, Broadmoor and Rampton), and a review team - chaired by Sir Richard Tilt, former Director of the English Prison Service - was formed. There was no clinician on the inquiry team. The terms of reference for the security review included 'a comprehensive review of all aspects of physical and relational security in each of the High Security Hospitals' as well as the identification of 'improvements required to security policies and practice in the three hospitals' and 'recommendations on any physical improvements required' (Tilt et al, 2000: Appendix 1).

It is contended that a fundamental lack of understanding of the concept of security, as reflected in the terms of reference, flawed the review from the outset. Moreover, the review team was inappropriately constituted and thus unable to consider properly the contribution each component makes to security in a therapeutic setting such as a high secure hospital.

\section{THE COMPONENTS OF SECURITY}

Faulk (1985) described his 'formula for a successful [hospital] secure unit' as including '(1) sufficient physical security appropriate to the patient; (2) high staff ratios; and (3) a therapeutic policy which encompasses individual programmes'. This encapsulates the three ingredients for successful security: a physical contribution, good staffing and appropriate policies.

The joint Department of Health and Home Office review of services for

†See letter, p. 548, this issue. mentally disordered offenders (the Reed Report) included a Working Group on High Security and Related Psychiatric Provision (Department of Health, 1994) which considered security in the special hospitals. Three components to security were defined, starting from 'the guiding principle...that the most effective form of security and, indeed, safety lies in the treatment of the patient'.

First, the concept of relational security is concerned with a detailed knowledge of the patients, their backgrounds and the reasons behind their admissions to a highsecurity hospital, and this comes from therapeutic relationships as well as the wider treatment programmes. Thus security is directly linked with treatment. Such work must be conducted within a safe environment and relational security is complemented by physical and procedural security. Physical security comprises the physical deterrents to escape from the hospital, such as locked doors, escape-proof windows and perimeter walls. The final part of the security triad is procedural security, which is described as 'the methodology or systems by which patients are managed and safe security maintained'. This includes, for example, operational procedures for screening staff and visitors to the hospital, searching patients, and also having safe staffing levels.

The distinction between relational, physical and procedural security is artificial. Security should be viewed as an indivisible whole; ignore or neglect one feature and security overall is weakened. This fact is acknowledged in Prison Service reports into disturbances at or escapes from prisons. The Learmont Inquiry (Home Office, 1995) into an escape from Parkhurst emphasised the importance of 'dynamic security'. This is akin to 'relational security' as it incorporates a sense of the prisoner as an individual as well as the relationships he or she develops with staff and other inmates. The Inquiry report went on to recognise that maintaining security (preventing prison escapes) and control (ensuring safety inside the prison) at prisons is 'only really possible through the relationship between staff and inmates' (Home Office, 1995: paragraph 5.55). The report commented on the need for 'having custody [or physical security], care [referred to elsewhere in the report as humanitarianism, or, above, as relational security] and control [procedural security] in harmony' (Home Office, 1995: paragraph 3.32).

The Woolf Report (Home Office, 1991), into disturbances at various prisons, recast the caring aspect (or humanitarianism) of incarceration in terms of 'justice': defined as the 'obligation to treat prisoners with humanity and fairness and prepare them for return to the community' (Home Office, 1991: paragraph 9.20). The Inquiry concluded that the prison disturbances of 1990 came about fundamentally because of 'the failure of the Prison Service to fulfil its responsibilities to act with justice' (Home Office, 1991: paragraph 9.24).

None the less, differences between secure hospitals and prisons do exist and this was unacknowledged in the Tilt Report. Prisoners are dealt with as groups and provided with 'regimes', whereas hospital patients need and receive much more individualised attention.

\section{RECOMMENDATIONS FROM THE SECURITY REVIEW OF HIGH SECURE HOSPITALS}

The Tilt review team made 50 general recommendations: three-quarters related to procedural security issues and a quarter to physical security (Tilt et al, 2000). Recommendations to increase the physical security at the high secure hospitals included enhancing perimeter security, changing to a magnetic locking system and examining 'the feasibility of locking the rooms at night of all patients on admission and intensive care wards and all "high risk” patients'. Recommendations for procedural security included enhancing the profile accorded to security itself within the hospitals: increasing the staffing levels in the security departments, developing 'an effective security intelligence system' at each hospital and having dedicated search teams.

In contrast, relational security is scarcely mentioned in the entire report 
although it was specifically referred to in the first of the terms of reference for the security review. The closest any of the recommendations came to addressing relational security is the call for 'a procedure...to identify "high risk" patients' (paragraph 7.5) but this is only to subject them to higher level (or 'special') 'procedural security arrangements' (paragraph 7.5) rather than assisting with their treatment or rehabilitation.

\section{CONSEQUENCES FOR SECURE PSYCHIATRIC SERVICES}

The Tilt Report acknowledged that for high-security hospitals, 'security and therapeutic issues [are] so closely interrelated that security could not, and should not, be dealt with in isolation' (Tilt et al, 2000: paragraph 2.3). One should go further because in high secure hospitals therapy in its widest sense is an integral part of security. Not to have considered therapeutic issues in their own right, as physical elements and procedural matters were, is a major omission of the review.

The emphasis throughout the report on the more tangible aspects of security such as high walls and better locks, and the virtual absence of consideration of the less overt contribution of relational security, fits in with the official preoccupation with 'dangerousness' in recent years. Recent evidence of the greater prominence of public protection within the mental health service provision is seen in initiatives such as the creation of a new 'condition' - dangerous severe personality disorder (DSPD) - which requires a new form of service provision and could permit preventive detention (Home Office \& Department of Health, 1999).

The Tilt Report acknowledged that 'there have been no escapes (since 1994) or absconsions from 1997 onwards... Nevertheless, the underlying dangerousness of the patient population and the potential threat which they represent to members of the general public, and to staff and fellow patients within the hospitals, should not be underestimated' (Tilt et al, 2000: paragraph 3.2). It is difficult to know whether the risks are underestimated (or overestimated), when they are not quantified in the report. Evidence from published research (although it was not included in the Tilt Report) reveals seven escapes from the high secure hospitals between 1976 and
1988, including two occasions when patients exited via the gate (Huws \& Shubsachs, 1993), and a further 12 breaches of physical security between 1989 and 1994 (Moore, 2000). Fourteen escapes from one particular high secure hospital between 1985 and 1996 occurred from sites other than the part of the hospital campus surrounded by the $6 \mathrm{~m}$ wall (Brook et al, 1999). Escapes from prison establishments are a recent addition to the annually produced prison statistics. There has been no escape from a Category B (closed training) prison since 1995, when there was one, although seven had occurred in 1994 (Home Office, 2000).

Another recommendation in the review's report, unimpeded by the lack of supporting evidence, was the feasibility be examined of locking patients in their rooms at night on particular wards (Tilt et al, 2000: paragraph 10.4). The policy of ' 24 hour care' was introduced into high secure hospitals from 1991 onwards in line with the Mental Health Act Code of Practice (Department of Health \& Welsh Office, 1999). As the review team acknowledged, it does not seem to have 'resulted in a higher number of night-time security incidents'; nevertheless, in their opinion this policy remains a 'potential threat to security'. The recommendation signals a significant shift from an ethos of care towards one of containment.

The White Paper Reforming the Mental Health Act (Department of Health \& Home Office, 2000: Part II, paragraph 6.23) highlighted the need for staff: 'where individuals are detained as a result of their mental disorder, they must be held in a therapeutic environment which is designed to address their needs effectively. This is not just a matter of new places... but also properly trained staff, new approaches to assessment and treatment and a rigorous programme of research and evaluation.' This view of managing detained patients contrasts sharply with the Tilt Report, which has no discussion about the impact of staffing.

Inquiry reports for the Prison Service have acknowledged the fundamental contribution that staff make to the security of an establishment. The Learmont Report was particularly forthright: 'The importance of able and dedicated staff....cannot be overemphasised. Whilst technology and physical barriers are an essential part of security, they will be of little use without the active commitment of fully motivated staff'
(Home Office, 1995: paragraph 3.3). The current negative public image of the high secure hospitals already has had an impact on the recruitment and retention of staff, as well as on the morale of those remaining. There will also be security consequences if staff members are unable to do the jobs they were originally trained for. Most obviously affected will be relational security, but the effects of poor staff morale can also have ramifications into physical security (Home Office, 1995: paragraph 4.39) and the way in which security procedures are operationalised.

The Tilt Report's recommendations, when implemented in full, as the Government has committed itself to doing, will produce an imbalance between the security and therapeutic objectives of high secure hospitals. Any immeasurable benefit from reducing further the incidence of newsworthy but rare events such as escapes will be at the expense of exacerbating the dehumanising effects of incarceration which, in the long run, will increase the very dangers the new measures are supposed to reduce. The Woolf Report spoke of attending to the humanity of detention and how 'excessive security and control can have the opposite effect to the ones desired. Prisoners will feel unnecessarily oppressed' (Home Office, 1991).

An immediate concern is that patients will be ill-equipped to move on to accommodation of lesser security and could consequently spend more time in a high secure setting. This would counteract the Government's plan to have fewer patients in high security. Rehabilitation programmes will be restricted and the value of parole (unescorted leave within the hospital perimeter) will be undermined when patients are not routinely escorted within the secure perimeter and physical security has been greatly enhanced. The gap between high and medium security will be widened to the point that medium secure units will have to choose between taking a patient in the hope that that patient has probably made the necessary progress, even though it cannot be adequately demonstrated, and not taking the patient at all.

For medium secure units the sting of the report is in its tail. One recommendation called for 'a nationally led review of medium secure provision' (Tilt et al, 2000). In part this is a review of the capacity of the medium secure estate in anticipation of further transfers from high 
secure hospitals. However, it will also address the capability of such units. Given the nature of the review in high security, it seems inevitable that 'capability' will be taken to mean the ability to contain patients within the units rather than successfully rehabilitate them for a return to living in the community.

\section{CONCLUSION}

It is salutary to reflect that the security review of high secure hospitals derived from the second Ashworth Inquiry (Fallon et al, 1999), which accused individuals of being ignorant of 'the proper balance of security and therapy'. Yet the Report (Tilt et al, 2000) is an incomplete consideration of the composite nature of security in a high-security hospital. Its recommendations have greatest impact on procedural aspects, but also include high-profile ideas such as additional perimeter security, which are difficult to justify on the evidence presented. The Report ignores completely the value of having adequate numbers of appropriately trained staff from all disciplines to enhance and develop therapeutic programmes and thereby improve relational security.

\section{ACKNOWLEDGEMENT}

We thank Dr Sophie Davison for her helpful comments and suggestions on earlier drafts of the paper.

TIM EXWORTHY, MRCPsych, Redford Lodge Hospital, Edmonton; JOHN GUNN, FRCPsych, Department of Forensic Psychiatry, Institute of Psychiatry, London, UK

Correspondence: DrTim Exworthy, Consultant Psychiatrist, Redford Lodge Hospital, 15 Church Street, Edmonton N9 9DY, UK

(First received 14 February 2002, final revision 27 August 2002, accepted 27 August 2002)

\section{DECLARATION OF INTEREST}

T.E. was a consultant forensic psychiatrist at Broadmoor Hospital (1995-1999) before taking up his current post at an independent medium secure unit run by Partnerships in Care. J.G. is currently Chairman of the Faculty of Forensic Psychiatry of the Royal College of Psychiatrists, but the views expressed here are his alone and do not necessarily represent those of the Faculty or the College.

\section{REFERENCES}

Brook, R., Dolan, M. \& Coorey, P. (1999) Absconding of patients detained in an English Special Hospital. Journal of Forensic Psychiatry, 10, 46-58.

Department of Health (1994) Report of the Working Group on High Security and Related Psychiatric Provision [the Reed Report]. London: Department of Health.

_ \& Home Office (2000) Reforming the Mental Health Act (CM 5016, I \& II). London: Stationery Office.

— \& Welsh Office (1999) Code of Practice: Mental Health Act 1983. London: Stationery Office.

Fallon, P., Bluglass, R., Edwards, B., et al (1999) Report of the Committee of Inquiry into the Personality
Disorder Unit, Ashworth Special Hospital (vol. I) (Cm 4194, II). London: Stationery Office.

Faulk, M. (1985) Secure facilities in local psychiatric hospitals. In Secure Provision (ed. L. Gostin). London: Tavistock.

Home Office (1991) Prison Disturbances April 1990. Report of an Inquiry (Cm 1456). London: HMSO.

- (1995) Review of Prison Service Security in England and Wales and the Escape from Parkhurst Prison on Tuesday 3rd January 1995 [the Learmont Inquiry]. London: $\mathrm{HMSO}$

- (2000) Prison Statistics England and Wales 1999 (Cm 4805). London: Stationery Office.

_ \& Department of Health (1999) Managing Dangerous People with Severe Personality Disorder: Proposals for Policy Development. London: Home Office/ Department of Health.

Huws, R. \& Shubsachs, A. (1993) A study of absconding by Special Hospital patients: 1976 to 1988 Journal of Forensic Psychiatry, 4, 45-58.

Moore, E. (2000) A descriptive analysis of incidents of absconding and escape from the English high-security hospitals, 1989-94. Journal of Forensic Psychiatry, II, 344-358.

Tilt, R., Perry, B., Martin, C., et al (2000) Report of the Review of Security at the High Security Hospitals. London: Department of Health. 